\title{
Editorial
}

\section{New strategies in plastic surgery: autologous adipose-derived mesenchymal stem cells contained in fat grafting improves symptomatic scars}

\author{
Pietro Gentile ${ }^{1,2, *}$ \\ ${ }^{1}$ Department of Surgical Science, “Tor Vergata” University, 00133 Rome, Italy, ${ }^{2}$ Academy of International Regenerative \\ Medicine \& Surgery Societies (AIRMESS), 1201 Geneva, Switzerland
}

\section{TABLE OF CONTENTS}

\author{
1. Introduction \\ 2. Treatment of symptomatic scars with fat grafting and AD-MSCs: background and procedures \\ 3. Symptomatic scar treatment with fat grafting and AD-MSCs: clinical and histological outcomes \\ 4. Symptomatic scar treatment with fat grafting and AD-MSCs: the role of age in the activity of $A D$-MSCs \\ 5. Conclusions \\ 6. Ethics approval and consent to participate \\ 7. Acknowledgment \\ 8. Funding \\ 9. Conflict of interest \\ 10. References
}

\section{Introduction}

Symptomatic scars represent a challenge for regenerative plastic surgeons. Several procedures based on a tissue engineering approach and different cellular products have been suggested, aiming to improve wound healing (WH) and scar therapy (ST). In the field of autologous regenerative strategies, the effectiveness of autologous adipose-derived mesenchymal stem cells (ADMSCs), contained in fat grafts (FG) has been evaluated in several fields, from facial rejuvenation to complex wound therapy [1]. The clinical improvements obtained by FG are related to AD-MSCs, contained in the stromal vascular fraction (SVF) [2], consisting of a mixture of pericytes, leukocytes, endothelial and smooth muscle cells [3]. Additionally, the FG contains several cells (AD-MSCs and adipocytes), extracellular matrix (ECM), nerves and vessels [3]. For the above-mentioned reasons, the FG may be considered both a biologically active tissue with regenerative properties when it is directly injected into the damaged tissue, and as a scaffold when it is enriched with ADMSCs [1, 2]. FGs may act as a scaffold for AD-MSCs, representing a biological matrix (cellular and extracellular) in which these cells can be incorporated and transported, resulting in improved healing time and scar signs and symptoms, via an autologous regenerative approach. The percentage of AD-MSC contained in SVF varies depending on the extraction procedure (enzymatic digestion vs mechani- cal manipulation based on centrifugation and filtration) but is greater than in a classic FG [4]. The present editorial aims to summarize and critique the most recent literature on scar signs/symptom resulting from FG and AD-MSCs, also considering the several different FG preparation procedures.

\section{Treatment of symptomatic scars with fat grafting and AD-MSCs: background and procedures}

Currently, ST presents several limits because in several cases does not sufficiently reduce scar visibility and related symptoms [5]. Scars result from the distinct steps of WH (i.e., inflammation, formation of new tissue and its remodeling), ending by fibrosis. The $\mathrm{WH}$ process results in a scar that can be indistinguishable from normal skin (normotrophic and physiological scar) or may acquire pathological features such as in the case of hypertrophic and keloid scars [6] showing more frequent clinical symptoms (symptomatic scars) [5, 6]. Scar visibility is a burden for the patient, due to differences in texture and color as compared to normal skin and/or for volume defects. Moreover, scars may be painful, itchy, and in certain cases cause functional impairment by movement restriction [5]. FG appears to be a promising therapy for ST because it improves soft tissue volume defects and improves scar quality e.g., elasticity $[7,8]$. 
In the last few years, new FG preparation procedures have been developed. In fact, FG has been used both as bioactive material through the lipostructure, nano-fat and micro-fat procedures, as a bioactive scaffold when it was enhanced with SVF acting as a direct source of AD-MSCs [1]. Micro-fat and nano-fat represent new promising techniques of lipofilling, especially in the treatment of superficial skin scars [4, 7]. Many procedures based on filtration, emulsification and centrifugation procedures have been reported to obtain micro-and nano-fat [9].

Additionally, FG is known to reduce neuropathy although the mechanism remains elusive. AD-MSCs might play a pivotal role as they also appear to reduce scar-related pain [10]. From a biological perspective, it remains elusive why FG leads to clinical improvements, including improvement of the aesthetic aspect, normalization of tissue elasticity, and reduction of scar-related pain.

For this reason, the present editorial aims to analyze the clinical results of FG and AD-MSCs on symptomatic scars and to better understand the biomolecular pathway that may explain its mechanism.

\section{Symptomatic scar treatment with fat grafting and AD-MSCs: clinical and histological outcomes}

Several studies have been published on the potential role of FG and their AD-MSCs on signs/symptoms of scar improvement.

In a recent investigation, Jaspers et al. [7] found improved skin elasticity in people who underwent a single FG. Similar clinical results were analyzed by Maione et al. [8], who observed that skin hardness decreased after lipofilling in scars. In contrast, Gal et al. [11], reported the absence of scar quality improvements after FG in burn scars. These pioneering investigations had several limitations represented by the absence of skin histology analysis, and the absence of correlation between findings and cell content in FG. Additionally, the evidence-based medicine (EBM) level of the studies analyzed and summarized in two systematic reviews [12, 13] and one literature review [14], was low. Thus, randomized controlled trials (EBM level I), to confirm clinical efficacy, in this specific field, are lacking to date. In a previous clinical investigation, histological changes in scar tissues after FG were incidentally reported. In a study performed by Bruno et al. [15], POSAS scores suggested scar improvement after FG in burn scars, which coincided with increased proliferation in the scar as well as changes in the ECM. Despite the interesting results analyzed, it was unclear whether histological examinations were carried out on biopsies of a single patient, or more patients. Klinger et al. [16] described an increase in vessel density and epithelial hyperplasia after FG in the scar tissue of a single patient. Additionally, the role of the SVF and its clinical implications in scars treatment, represented by an improvement of scar tissue quality and related soft tissue volume, has been discussed in several studies [17, 18].

Here, we suggest that significant clinical improvement of symptomatic scars is associated with histological changes. Following this concept, multiple FG treatments would offer a better outcome compared to a single procedure. In fact, in the analyzed studies [3, 7, 17, 18] an increase in epidermal proliferation and vessel density was observed after the second FG, and remodeling of ECM structure occurred three months after the last treatment. The ECM in typical scar tissue, consisting of thick, highly aligned fibrils, was replaced by or transformed into thinner, smaller bundles with a more typical physiological organization. These changes were most obvious in highly vascularized areas, where the action of $\mathrm{T}$ lymphocytes, mast cells, and M2 macrophages had taken place. Thus, changes in immune balance may play an important role in the observed pro-regenerative effect of FG. To date, limited studies in vitro and in vivo, suggest both a stimulating as well as a suppressing effect of M2 macrophages and mast cells on scarring, which depends on time and local microenvironmental conditions [19-21].

The polarization of macrophages seems to play an essential role in wound healing. Interestingly, a recent report demonstrated that extracellular vesicles from human MSCs have anti-inflammatory properties as they prevent the polarization of macrophages into the pro-inflammatory M1 phenotype [22].

\section{Symptomatic scar treatment with fat grafting and AD-MSCs: the role of age in the activity of AD-MSCs}

It remains to be clarified whether the patient's age may impact the beneficial effect and neuroectodermal properties of the autologous AD-MSCs. In this respect, it is known that there is a decline in the function of bone marrow-derived MSCs with increasing donor age which may limit the use of autologous transplantation [23]. According to Hermann et al. [23], the neuroectodermal differentiation potential of MSCs derived from old donors is completely lost. Comparison of young versus old donorderived MSCs showed fewer cells expressing early neuroectodermal marker proteins in the older samples. In the study of Hermann et al. [23], qRT-PCR showed reduced expression of the proliferation marker Ki67 and the neuroectodermal gene NES (Nestin) in old donor-derived cells compared with young donor MSCs [23]. Their data provide evidence for senescence and reduced multipotentiality of old donor-derived MSCs. 


\section{Conclusions}

Despite average-quality evidence, the analyzed data provide indications of positive effects of FG and ADMSCs in signs/symptoms of scars-both clinically and on a microscopic level. The induction of a pro-regenerative immune response increases vascularization, with epidermal proliferation and remodeling of scar tissue extracellular matrix being the proposed biomolecular mechanisms.

\section{Ethics approval and consent to participate}

Not applicable.

\section{Acknowledgment}

Not applicable.

\section{Funding}

This research received no external funding.

\section{Conflict of interest}

The author declares no conflict of interest.

\section{References}

[1] Gentile P, Sterodimas A, Calabrese C, Garcovich S. Systematic review: Advances of fat tissue engineering as bioactive scaffold, bioactive material, and source for adipose-derived mesenchymal stem cells in wound and scar treatment. Stem Cell Research \& Therapy. 2021; 12: 318.

[2] Gentile P, Garcovich S. Systematic Review: Adipose-Derived Mesenchymal Stem Cells, Platelet-Rich Plasma and Biomaterials as New Regenerative Strategies in Chronic Skin Wounds and Soft Tissue Defects. International Journal of Molecular Sciences. 2021; 22: 1538.

[3] Cervelli V, Gentile P. Use of cell fat mixed with platelet gel in progressive hemifacial atrophy. Aesthetic Plastic Surgery. 2009; 33: 22-27.

[4] Trivisonno A, Alexander RW, Baldari S, Cohen SR, Di Rocco G, Gentile P, et al. Intraoperative Strategies for Minimal Manipulation of Autologous Adipose Tissue for Cell- and Tissue-Based Therapies: Concise Review. Stem Cells Translational Medicine. 2019; 8: 1265-1271.

[5] Aarabi S, Longaker MT, Gurtner GC. Hypertrophic scar formation following burns and trauma: new approaches to treatment. PLoS Medicine. 2007; 4: e234.

[6] van der Veer WM, Bloemen MCT, Ulrich MMW, Molema G, van Zuijlen PP, Middelkoop E, et al. Potential cellular and molecular causes of hypertrophic scar formation. Burns. 2009; 35: 15-29.

[7] Jaspers MEH, Brouwer KM, van Trier AJM, Groot ML, Middelkoop E, van Zuijlen PPM. Effectiveness of Autologous Fat Grafting in Adherent Scars. Plastic and Reconstructive Surgery. 2017; 139: 212-219.

[8] Maione L, Memeo A, Pedretti L, Verdoni F, Lisa A, Bandi V, et al. Autologous fat graft as treatment of post short stature surgical correction scars. Injury. 2014; 45: S126-S132.

[9] Gentile P, Calabrese C, De Angelis B, Pizzicannella J, Kothari A, Garcovich S. Impact of the Different Preparation Methods to
Obtain Human Adipose-Derived Stromal Vascular Fraction Cells (AD-SVFs) and Human Adipose-Derived Mesenchymal Stem Cells (AD-MSCs): Enzymatic Digestion Versus Mechanical Centrifugation. International Journal of Molecular Sciences. 2019; 20: 5471 .

[10] Huang S, Wu S, Chang K, Lin C, Chang C, Wu Y, et al. Alleviation of neuropathic scar pain using autologous fat grafting. Annals of Plastic Surgery. 2015; 74: S99-104.

[11] Gal S, Ramirez JI, Maguina P. Autologous fat grafting does not improve burn scar appearance: a prospective, randomized, double-blinded, placebo-controlled, pilot study. Burns. 2017; 43: 486-489.

[12] Negenborn VL, Groen J, Smit JM, Niessen FB, Mullender MG. The Use of Autologous Fat Grafting for Treatment of Scar Tissue and Scar-Related Conditions: a Systematic Review. Plastic and Reconstructive Surgery. 2016; 137: 31e-43e.

[13] Condé-Green A, Marano AA, Lee ES, Reisler T, Price LA, Milner SM, et al. Fat Grafting and Adipose-Derived Regenerative Cells in Burn Wound Healing and Scarring: a Systematic Review of the Literature. Plastic and Reconstructive Surgery. 2016; 137: 302312.

[14] Spiekman M, van Dongen JA, Willemsen JC, Hoppe DL, van der Lei B, Harmsen MC. The power of fat and its adipose-derived stromal cells: emerging concepts for fibrotic scar treatment. Journal of Tissue Engineering and Regenerative Medicine. 2017; 11: 3220-3235.

[15] Bruno A, Delli Santi G, Fasciani L, Cempanari M, Palombo M, Palombo P. Burn scar lipofilling: immunohistochemical and clinical outcomes. Journal of Craniofacial Surgery. 2013; 24: 18061814.

[16] Klinger M, Marazzi M, Vigo D, Torre M. Fat injection for cases of severe burn outcomes: a new perspective of scar remodeling and reduction. Aesthetic Plastic Surgery. 2008; 32: 465-469.

[17] Gentile P, Scioli MG, Bielli A, Orlandi A, Cervelli V. Comparing different nanofat procedures on scars: role of the stromal vascular fraction and its clinical implications. Regenerative Medicine. 2017; 12: 939-952.

[18] Gentile P, De Angelis B, Pasin M, Cervelli G, Curcio CB, Floris $\mathrm{M}$, et al. Adipose-derived stromal vascular fraction cells and platelet-rich plasma: basic and clinical evaluation for cell-based therapies in patients with scars on the face. Journal of Craniofacial Surgery. 2014; 25: 267-272.

[19] Ploeger DT, Hosper NA, Schipper M, Koerts JA, de Rond S, Bank RA. Cell plasticity in wound healing: paracrine factors of M1/M2 polarized macrophages influence the phenotypical state of dermal fibroblasts. Cell Communication and Signaling. 2013; 11: 29.

[20] Niessen FB, Schalkwijk J, Vos H, Timens W. Hypertrophic scar formation is associated with an increased number of epidermal Langerhans cells. Journal of Pathology. 2004; 202: 121-129.

[21] Beer TW, Baldwin H, West L, Gallagher PJ, Wright DH. Mast cells in pathological and surgical scars. British Journal of Ophthalmology. 1998; 82: 691-694.

[22] Pacienza N, Lee RH, Bae E, Kim D, Liu Q, Prockop DJ, et al. In Vitro Macrophage Assay Predicts the in Vivo Anti-inflammatory Potential of Exosomes from Human Mesenchymal Stromal Cells. Molecular Therapy. Methods \& Clinical Development. 2018; 13: 67-76.

[23] Hermann A, List C, Habisch H, Vukicevic V, Ehrhart-Bornstein M, Brenner R, et al. Age-dependent neuroectodermal differentiation capacity of human mesenchymal stromal cells: limitations for autologous cell replacement strategies. Cytotherapy. 2010; 12: 17-30.

Send correspondence to: Pietro Gentile, Department of Surgical Science, “Tor Vergata” University, 00133 Rome, Italy, Academy of International Regenerative Medicine \& Surgery Societies (AIRMESS), 1201 Geneva, Switzerland E-mail: pietrogentile2004@libero.it 